\title{
Uso de simuladores virtuais no ensino de Enfermagem: scoping review
}

\author{
Use of virtual simulators in the teaching of Nursing: scoping review \\ Uso de simuladores virtuales en la enseñanza de Enfermería: scoping review
}

Recebido: 25/02/2021 | Revisado: 03/03/2021 | Aceito: 04/03/2021 | Publicado: 12/03/2021

\author{
Michele Antunes \\ ORCID: https://orcid.org/0000-0002-7231-8099 \\ Universidade Feevale, Brasil \\ E-mail: micheleantunes@gmail.com \\ Cátia Aguiar Lenz \\ ORCID: https://orcid.org/0000-0003-1864-6988 \\ Universidade Feevale, Brasil \\ E-mail: lenz@feevale.br \\ Claudir Lopes da Silva \\ ORCID: https://orcid.org/0000-0001-8751-3199 \\ Universidade Feevale, Brasil \\ E-mail: claudir@feevale.br \\ Raquel de Lima dos Santos \\ ORCID: https://orcid.org/0000-0002-2891-0052 \\ Universidade Feevale, Brasil \\ E-mail: raquel.dlds@gmail.com \\ Marta Rosecler Bez \\ ORCID: https://orcid.org/0000-0002-5542-8229 \\ Universidade Feevale, Brasil \\ E-mail: martabez@gmail.com
}

\begin{abstract}
Resumo
O uso da simulação virtual pode proporcionar um ambiente seguro e de qualidade para o desenvolvimento das competências essenciais dos estudantes de enfermagem. O objetivo foi identificar a produção científica sobre o uso de simuladores virtuais no ensino de Enfermagem. Trata-se de um scoping review, realizado na Web of Science, Scopus, Pubmed/Medline, SciELO e BDENF, no período de 2005 a 2020. A amostra foi composta de 14 artigos. A análise permitiu identificar a prevalência de estudos nas áreas da saúde da criança e segurança do paciente, e o uso de "Simulação - paciente virtual". Os simuladores virtuais podem contribuir no ensino na enfermagem, pois permitem que o estudante coloque em prática os conhecimentos da teoria, vivenciando situações clínicas, o que pode auxiliar no desenvolvimento do pensamento crítico.
\end{abstract}

Palavras-chave: Enfermagem; Ensino; Simulação por computador.

\begin{abstract}
The use of virtual simulation can offer a safe and quality environment for the development of essential skills of nursing students, during the clinical decision-making process. The general objective was to identify the scientific production on the use of virtual simulators in nursing education. This is a scoping review, carried out on the Web of Science, Scopus, Pubmed/Medline, SciELO e BDENF, in the period from 2005 to 2020. The sample consisted of 14 articles. The analysis allowed to identify the prevalence of studies in the areas of child health and patient safety, and the use of "Simulation - virtual patient". Virtual simulators can contribute to the teaching of nursing, as they can help the student to practice the knowledge of theory, clinical experiences, or that can assist in the development of critical thinking.
\end{abstract}

Keywords: Nursing; Teachin; Computer simulation.

\section{Resumen}

El uso de la simulación virtual puede ofrecer un entorno seguro y de calidad para el desarrollo de habilidades esenciales de los estudiantes de enfermería, durante el proceso de toma de decisiones clínicas. El objetivo general fue identificar la producción científica sobre el uso de simuladores virtuales en la educación de enfermería. Esta es una scoping review, realizada en las bases de datos Web of Science, Scopus, Pubmed/Medline (Medical Literature Analysis and Retrieval, SciELO (Scientific Electronic Library Online) e BDENF (Base de Dados de Enfermagem), en el período comprendido entre 2005 y 2020. La muestra consistió en 14 artículos. El análisis permitió identificar la prevalencia de los estudios en las áreas de salud infantil y seguridad del paciente, y el uso de "Simulación - paciente virtual". Los simuladores virtuales pueden contribuir a la enseñanza de enfermería, ya que pueden ayudar al estudiante a practicar el conocimiento de la teoría, las experiencias clínicas o que pueden ayudar en el desarrollo del pensamiento crítico.

Palabras clave: Enfermería; Enseñando; Simulación por Computador. 


\section{Introdução}

As tecnologias educacionais digitais (TED) contribuem ao ensino de habilidades de enfermagem, melhorando a aquisição de referenciais teóricos que subsidiam as práticas, tendo em vista que as modalidades híbridas de ensino, que integrem TED e manequins em ambientes simulados, proporcionam métodos flexíveis que desenvolvem o pensamento crítico e priorizam o protagonismo do estudante (Silveira \& Cogo, 2017). E no ensino atual, vê-se a necessidade de incorporar estas tecnologias inovadoras ao ensino como uma necessidade real nos ambientes de educação (Mendonça, 2019).

A grande diferença entre uma prática específica de um procedimento para um momento de simulação consiste no fato de que este mesmo procedimento estará contextualizado, isto é, o aluno já deve deter o conhecimento técnico do procedimento realizado no ambiente, e agora ele precisará entender quando deverá realizá-lo em ambiente contextualizado (Melo, et al., 2017). A simulação pode gerar benefícios importantes para o ensino na Enfermagem ao aproximar a teoria da prática, a atender aos objetivos propostos, desenvolvimento do raciocínio diagnóstico e julgamento clínico, além do desenvolvimento de competências, habilidades e atitudes no ensino, assistência, pesquisa e gestão (Rodrigues, et al., 2020). Dentre estas ferramentas, destacam-se o uso das simulações virtuais.

A utilização desse tipo de simulação tem como vantagem o uso de um formalismo que permite a criação de simulações nas quais o professor não necessita conhecer informática para o desenvolvimento do material pedagógico. O foco está na criação dos casos clínicos, e não no conhecimento de programação ou tarefas complexas de informática (Bez, et al., 2018). A simulação virtual poderá proporcionar mais segurança e qualidade durante o processo de tomada de decisão clínica dos estudantes de enfermagem, a partir do desenvolvimento do pensamento crítico e raciocínio clínico (Antunes, 2020).

Estes variados tipos de simuladores podem ser utilizados a fim de simular situações clínicas manifestadas pelos pacientes, e a simulação desta, possibilita que os alunos experienciem tais cenários antes que sejam vivenciados na prática assistencial. Diante do exposto, este estudo tem como objetivo geral identificar a produção científica sobre o uso de simuladores virtuais no ensino de enfermagem.

\section{Metodologia}

A metodologia deste estudo trata-se de uma scoping review, com a análise qualitativa dos estudos identificados, descrita pelos autores Arksey e O’Malley (2005), e atualizada por Levac et al (2010).

Para Pereira et al (2018), em estudos qualitativos emprega-se a metodologia da pesquisa-ação, a qual busca evidenciar nas literaturas pertinentes meios para a resolução do problema exposto, a partir da prática reflexiva a qual se investiga através da leitura, busca e, posteriormente, aplicação dessas informações para obtenção e análise dos dados. Para isso, se torna necessário compreender a utilização das normas exigidas de acordo com o tipo de estudo. E as revisões de escopo configuramse como uma ferramenta ideal para determinar a cobertura de literatura sobre um determinado tópico, indicando de maneira clara, o volume de literatura e estudos disponíveis, bem como, uma visão geral (ampla ou detalhada) (Munn, et al., 2018).

Dessa forma, as revisões de escopo objetivam examinar evidências emergentes, pois o seu foco não é esclarecer questões específicas, nem atribuir valor ao estudo, pois estes aspectos diferenciam-na da revisão sistemática. As principais diferenças entre a revisão sistemática e a scoping são: o problema de pesquisa é amplo, em vez de ser focado como na revisão sistemática; os critérios de inclusão/exclusão podem ser estabelecidos posteriormente, ao início da revisão; a escolha dos estudos não foca na qualidade da investigação dos artigos da amostra; pode ou não envolver extração de dados; a síntese é fundamentalmente qualitativa e raramente quantitativa; é utilizada para identificar as variáveis e as lacunas existente na literatura (Armstrong, et al., 2011).

As etapas realizadas para o desenvolvimento deste estudo estruturadas com base no referencial de Arksey \& O’Malley (2005) e Levac et al (2010), quais sejam: identificação da questão de pesquisa; identificação de estudos relevantes; seleção dos 
estudos; extração de dados; separação, sumarização e relatório de resultados; e comunicação dos resultados.

Para construção da pergunta de pesquisa, utilizou-se a estratégia Population, Concept e Context (PCC) para uma scoping review (JBI, 2015), foram definidos: P- artigos; C- simulador virtual e C- ensino da enfermagem. A questão de pesquisa foi: "qual a produção científica sobre o uso de simuladores virtuais no ensino de enfermagem?" Para a identificação dos artigos, foram realizadas pesquisas nas bases de dados/repositórios de busca: Web of Science, Scopus, Pubmed/Medline (Medical Literature Analysis and Retrieval, SciELO (Scientific Electronic Library Online) e BDENF (Base de Dados de Enfermagem). As palavras-chave "simulação virtual" e "enfermagem" foram combinados com o operador boleano "AND", nos idiomas inglês e português.

Seguindo o referencial metodológico adotado, a scoping review preconiza que todos os estudos sejam inicialmente incluídos, para que após esta seleção, eles sejam avaliados pelo pesquisador. Para a inclusão dos estudos, os critérios de inclusão foram estabelecidos: artigos oriundos de estudos quantitativos, qualitativos e quanti-quali, relatos de experiência, estudos de caso, publicados em inglês e português, disponíveis online por meio de livre acesso, que atendessem a temática em estudo sobre o uso de simulação virtual no ensino da enfermagem, publicados no período de 2005 a 2020. Os critérios de exclusão foram: artigos de revisão e literatura cinzenta.

As buscas foram realizadas no mês de maio a junho de 2020. Após a aplicação dos critérios de inclusão/exclusão, os pesquisadores verificaram a existência de duplicidade nas bases de dados e realizam a leitura prévia do título e do resumo dos artigos. Neste momento, os estudos incluídos passam a ser lidos na íntegra, na busca por responder à questão norteadora.

$\mathrm{Na}$ etapa de separação, sumarização e relatório dos elementos essenciais encontrados em cada artigo, as pesquisadoras elaboraram um instrumento de coleta de dados, que permitiu a síntese, interpretação e análise dos achados de cada estudo. Informações como autoria, base/repositório de dados, local do estudo, ano de publicação, periódico, local de realização do estudo, características do simulador virtual e habilidades desenvolvidas, foram agrupados.

$\mathrm{Na}$ etapa final, por meio de uma categorização temática, organizada de acordo com as informações extraídas dos artigos, foi realizada a compilação e comunicação dos resultados, com a intenção de apresentar a visão geral de todo o material.

\section{Resultados e Discussão}

Dos 54 estudos encontrados, após leitura exaustiva dos títulos e resumos dos artigos, 10 artigos foram excluídos por estarem publicados em mais de uma base de dados, sendo contabilizado na primeira base. Entre estes, 44 foram selecionados para a leitura do título e resumo, e 23 foram excluídos por não atenderem a temática em estudo. Dos 21 artigos restantes, 07 foram excluídos por tratarem-se de estudos com a metodologia de revisão, compondo a amostra de 14 estudos selecionados.

Para elucidar o processo de busca, de exclusão e de seleção dos estudos que compuseram a amostra desta pesquisa, foi elaborado um Fluxograma (Figura 1), conforme recomendações do JBI, segundo checklist adaptado do Preferred Reporting Items for Systematic Reviews and Meta-Analyses (PRISMA) (Moher, et al., 2009). 
Figura 1 - Prisma sobre o processo de busca, exclusão e seleção dos estudos encontrados.

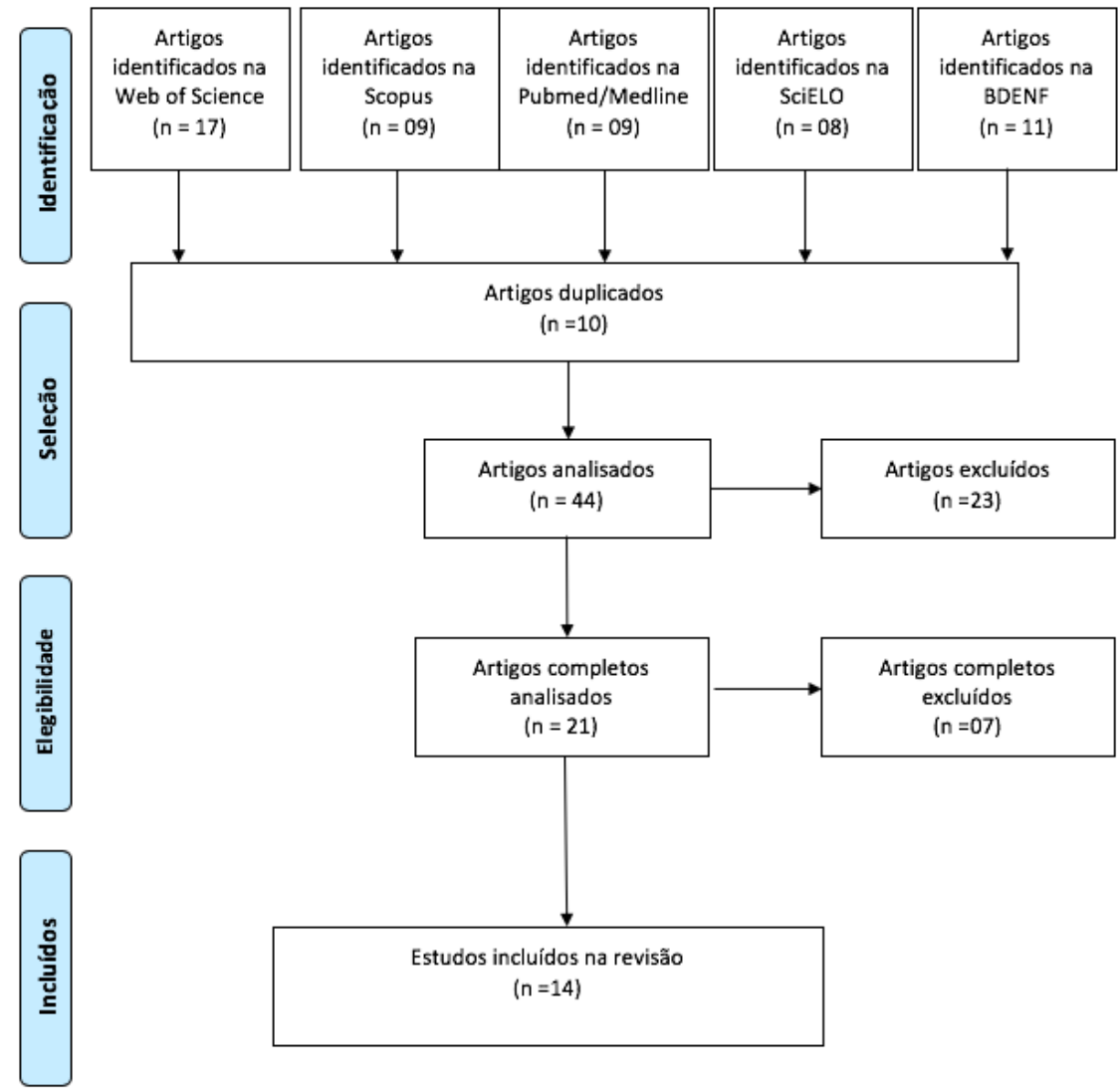

Fonte: Autores (2020). Modelo adaptado do fluxograma PRISMA.

A figura acima tem o objetivo de apresentar de maneira eficiente as etapas de seleção dos estudos que compuseram a amostra desta pesquisa, com as fases de identificação dos artigos nas bases de dados; seleção destes, com a exclusão daqueles que estavam duplicados; elegibilidade, com aqueles preenchiam os critérios de inclusão e exclusão; e a inclusão dos estudos analisados.

Os 14 estudos inclusos nesta revisão foram publicados no período de 2016 a 2020. Os estudos abrangeram abordagem quantitativa, qualitativa e quanti-qualitativos. Dentre eles, houve a prevalência de artigos científicos com a metodologia quantitativa $(07-50 \%)$, cinco $(35,7 \%)$ foram publicados no ano de 2018 , cinco $(35,7 \%)$ nas bases de dados Web os Science e Pubmed/MEDLINE (Quadro 1).

Quadro 1 - Caracterização dos estudos que compuseram a amostra deste estudo.

\begin{tabular}{|l|l|l|l|l|}
\hline Estudo & $\begin{array}{l}\text { Tipo de } \\
\text { estudo }\end{array}$ & Autoria/Ano & Título & $\begin{array}{l}\text { Base/Repositórios } \\
\text { dados }\end{array}$ \\
\hline 1 & Quantitativo & $\begin{array}{l}\text { Berg \& Steinsbekk } \\
(2020)\end{array}$ & $\begin{array}{l}\text { A prática individual em um aplicativo } \\
\text { de realidade virtual imersiva e } \\
\text { interativa é inferior à prática com } \\
\text { equipamentos tradicionais na } \\
\text { aprendizagem da observação clínica } \\
\text { sistemática? Um estudo controlado } \\
\text { randomizado }\end{array}$ & \\
\hline
\end{tabular}


Research, Society and Development, v. 10, n. 3, e20710313309, 2021

(CC BY 4.0) | ISSN 2525-3409 | DOI: http://dx.doi.org/10.33448/rsd-v10i3.13309

\begin{tabular}{|c|c|c|c|c|}
\hline 2 & $\begin{array}{l}\text { Quanti- } \\
\text { qualitativo }\end{array}$ & Daher et al. (2020) & O Simulador Físico-Virtual do Paciente & Web of Science \\
\hline 3 & Quantitativo & $\begin{array}{l}\text { Souza-Junior et al. } \\
(2020)\end{array}$ & $\begin{array}{l}\text { VIDA-Enfermagem v1.0: realidade } \\
\text { virtual imersiva na coleta de sangue a } \\
\text { vácuo em adulto }\end{array}$ & Web of Science \\
\hline 4 & Quantitativo & $\begin{array}{l}\text { Padilha et al. } \\
\text { (2019) }\end{array}$ & $\begin{array}{l}\text { Simulação virtual clínica no ensino de } \\
\text { enfermagem: ensaio clínico } \\
\text { randomizado }\end{array}$ & Web of Science \\
\hline 5 & Quantitativo & Polivka et al. (2019) & $\begin{array}{l}\text { Eficácia e usabilidade de um sistema } \\
\text { de treinamento em simulação virtual } \\
\text { para riscos à saúde e segurança } \\
\text { encontrado por trabalhadores da saúde }\end{array}$ & Web of Science \\
\hline 6 & Quantitativo & $\begin{array}{l}\text { Olszewski et al. } \\
(2018)\end{array}$ & $\begin{array}{l}\text { Ensino global de diálise peritoneal } \\
\text { pediátrica através de simulação virtual }\end{array}$ & Scopus \\
\hline 7 & $\begin{array}{l}\text { Quanti- } \\
\text { qualitativo }\end{array}$ & $\begin{array}{l}\text { Aebersold et al. } \\
(2018)\end{array}$ & $\begin{array}{l}\text { Treinamento interativo em simulação } \\
\text { virtual aumentada em anatomia }\end{array}$ & Pubmed/MEDLINE \\
\hline 8 & Quantitativo & $\begin{array}{l}\text { Tschannen et al. } \\
(2018)\end{array}$ & $\begin{array}{l}\text { Melhorar o conhecimento e o } \\
\text { comportamento da liderança e } \\
\text { seguidores entre a equipe } \\
\text { interprofissional }\end{array}$ & Pubmed/MEDLINE \\
\hline 9 & Qualitativo & Slater et al. (2019) & $\begin{array}{l}\text { Treinamento em simulação virtual: } \\
\text { experiência visual de demência }\end{array}$ & Pubmed/MEDLINE \\
\hline 10 & $\begin{array}{l}\text { Quanti- } \\
\text { qualitativo }\end{array}$ & Tjoflat et al. (2018) & $\begin{array}{l}\text { Estudantes } \quad \text { de } \quad \text { enfermagem } \\
\text { noruegueses: avaliação do vSim }{ }^{\circledR} \text { para } \\
\text { Enfermagem }\end{array}$ & Pubmed/MEDLINE \\
\hline 11 & Qualitativo & $\begin{array}{l}\text { Domingues et al. } \\
(2017)\end{array}$ & $\begin{array}{l}\text { Simulação virtual por computador no } \\
\text { ensino de enfermagem: relato de } \\
\text { experiência }\end{array}$ & BDENF \\
\hline 12 & $\begin{array}{l}\text { Quanti- } \\
\text { qualitativo }\end{array}$ & Jutten et al. (2017) & $\begin{array}{l}\text { Testando a efetividade do treinamento } \\
\text { misto de realidade virtual Into D'mentia } \\
\text { para cuidadores informais de pessoas } \\
\text { com demência: protocolo para um } \\
\text { estudo longitudinal, quase- } \\
\text { experimental }\end{array}$ & Scopus \\
\hline 13 & Quantitativo & Aredes et al. (2017) & $\begin{array}{l}\text { E-baby integridade da pele: inovação } \\
\text { tecnológica no ensino de enfermagem } \\
\text { neonatal baseado em evidências }\end{array}$ & SciELO \\
\hline 14 & $\begin{array}{l}\text { Quanti- } \\
\text { qualitativo }\end{array}$ & $\begin{array}{l}\text { Darragh et al. } \\
(2016)\end{array}$ & $\begin{array}{l}\text { Simulação de jogos como treinamento } \\
\text { em saúde e segurança para } \\
\text { profissionais de saúde em casa }\end{array}$ & Pubmed/MEDLINE \\
\hline
\end{tabular}


Fonte: Autores (2020).

O quadro acima tem o intuito de apresentar os artigos que compuseram a amostra, destacando o tipo de estudo, autoria, ano de publicação, título e base de dados no qual ele foi encontrado. Caso o mesmo artigo estivesse em mais de uma base de dados, ele era computado na base em que ele foi identificado primeiro.

O Quadro 2 apresenta o país no qual o estudo foi desenvolvido, a temática que foi o foco da simulação, características dos simuladores identificados e os objetivos dos estudos. Os Estados Unidos da América foi o país prevalente quanto a publicação de estudos na área de simuladores virtuais, com 06 (42,8\%) das pesquisas provenientes deste local. As temáticas realizadas durante as simulações foram variadas, com destaque para estudos que realizaram suas pesquisas na área da saúde da criança, e segurança do paciente, ambas com três $(21,4 \%)$ artigos.

Os objetivos dos estudos também foram variados, pois buscavam investigar, descrever, construir ou relatar a experiência do uso de simuladores no ensino de enfermagem. Destaca-se que nem todos os estudos atribuíram um nome para o simulador utilizado. As variáveis tais como, programação e o aprofundamento quanto ao design destas ferramentas, não foram objetos de análise deste artigo.

Quadro 2 - Estudos encontrados conforme ano de publicação, autoria, periódico/instituição, título, país do estudo e tipo de publicação

\begin{tabular}{|c|c|c|c|}
\hline Estudo & País do estudo & Temática da simulação & Objetivos dos estudos \\
\hline 1 & Noruega & $\begin{array}{l}\text { Avaliação do paciente no } \\
\text { pré-hospitalar }\end{array}$ & $\begin{array}{l}\text { Investigar se a auto-prática individual da abordagem vias } \\
\text { aéreas, respiração, circulação, incapacidade e exposição, em } \\
\text { um aplicativo de realidade virtual imersiva e interativa } \\
\text { proporcionou resultados de aprendizagem não inferiores em } \\
\text { comparação ao uso de equipamentos tradicionais. }\end{array}$ \\
\hline 2 & $\begin{array}{l}\text { Estados Unidos da } \\
\text { América }\end{array}$ & $\begin{array}{l}\text { Avaliação na área da } \\
\text { Saúde da criança }\end{array}$ & $\begin{array}{l}\text { Descrever a implementação da tecnologia, testes técnicos com } \\
\text { especialistas em saúde e um conselho de revisão institucional - } \\
\text { experimento piloto aprovado, envolvendo estudantes de } \\
\text { enfermagem interagindo com uma criança simulada em } 2 \\
\text { cenários: sepse e abuso infantil. }\end{array}$ \\
\hline 3 & Brasil & $\begin{array}{l}\text { Procedimento de } \\
\text { Enfermagem }\end{array}$ & $\begin{array}{l}\text { Desenvolver e validar a primeira versão do simulador de } \\
\text { realidade virtual imersiva no procedimento de coleta de sangue } \\
\text { a vácuo no paciente adulto - VIDA (Virtual Interactive } \\
\text { Distance-Learning on Anatomy)-Enfermagem v1.0. }\end{array}$ \\
\hline 4 & Portugal & $\begin{array}{c}\text { Avaliação do paciente } \\
\text { adulto }\end{array}$ & $\begin{array}{l}\text { Avaliar o efeito da simulação virtual clínica no que diz respeito } \\
\text { à retenção de conhecimento, raciocínio clínico, autoeficácia e } \\
\text { satisfação com a experiência de aprendizagem entre estudantes } \\
\text { de enfermagem. }\end{array}$ \\
\hline 5 & $\begin{array}{c}\text { Estados Unidos da } \\
\text { América }\end{array}$ & $\begin{array}{l}\text { Segurança do paciente na } \\
\text { atenção domiciliar }\end{array}$ & $\begin{array}{l}\text { Avaliar a eficácia, usabilidade, utilidade e conveniência de um } \\
\text { Sistema de Treinamento em Simulação Virtual Interativo em } \\
\text { Assistência Médica Domiciliar, projetado para treinar } \\
\text { profissionais de saúde domiciliar e estudantes de saúde para } \\
\text { identificar e responder a riscos de saúde e segurança nas casas } \\
\text { dos pacientes. }\end{array}$ \\
\hline
\end{tabular}


Research, Society and Development, v. 10, n. 3, e20710313309, 2021

(CC BY 4.0) | ISSN 2525-3409 | DOI: http://dx.doi.org/10.33448/rsd-v10i3.13309

\begin{tabular}{|c|c|c|c|}
\hline 6 & $\begin{array}{l}\text { Estados Unidos da } \\
\text { América }\end{array}$ & $\begin{array}{l}\text { Avaliação na área da } \\
\text { Saúde da criança }\end{array}$ & $\begin{array}{l}\text { Descrever o desenvolvimento de um simulador virtual de } \\
\text { diálise peritoneal e relatar os primeiros meses de uso online. }\end{array}$ \\
\hline 7 & $\begin{array}{l}\text { Estados Unidos da } \\
\text { América }\end{array}$ & $\begin{array}{l}\text { Estudo de anatomia/ } \\
\text { Procedimentos de } \\
\text { enfermagem }\end{array}$ & $\begin{array}{l}\text { Descrever os resultados sobre a capacidade de realizar a } \\
\text { passagem de sonda nasogástrica após serem aleatoriamente } \\
\text { designados para o treinamento habitual ou para um módulo de } \\
\text { treinamento em simulação virtual. }\end{array}$ \\
\hline 8 & $\begin{array}{l}\text { Estados Unidos da } \\
\text { América }\end{array}$ & Gestão em saúde & $\begin{array}{l}\text { Examinar o treinamento virtual sobre os princípios de liderança } \\
\text { e Gerenciamento de Recursos e de Pessoas. }\end{array}$ \\
\hline 9 & Irlanda & Saúde do Idoso & $\begin{array}{l}\text { Explorar o impacto de uma experiência de treinamento } \\
\text { interativo nos elementos morais, emotivos, comportamentais e } \\
\text { cognitivos da empatia. }\end{array}$ \\
\hline 10 & Noruega & $\begin{array}{l}\text { Cuidados de enfermagem } \\
\text { ao paciente cirúrgico }\end{array}$ & $\begin{array}{l}\text { Avaliar as experiências dos estudantes de enfermagem } \\
\text { noruegueses com o cenário virtual de simulação clínica em } \\
\text { enfermagem cirúrgica do } v \operatorname{Sim}{ }^{\circledR} \text { for Nursing. }\end{array}$ \\
\hline 11 & Brasil & Segurança do paciente & $\begin{array}{l}\text { Relatar a experiência da utilização de uma simulação virtual } \\
\text { por computador no ensino de segurança do paciente para } \\
\text { alunos de graduação em enfermagem. }\end{array}$ \\
\hline 12 & Holanda & Saúde do Idoso & $\begin{array}{l}\text { Avaliar a efetividade do simulador Into D'mentia em várias } \\
\text { variáveis ao longo do tempo, incluindo empatia, carga de } \\
\text { cuidador, sentimentos de competência em cuidar, depressão e } \\
\text { ansiedade, relação entre cuidadores e seus pacientes e saúde } \\
\text { dos cuidadores. }\end{array}$ \\
\hline 13 & Brasil & Saúde da criança & $\begin{array}{l}\text { Desenvolver e validar o serious game e-Baby: integridade da } \\
\text { pele junto a um painel de experts. }\end{array}$ \\
\hline 14 & $\begin{array}{l}\text { Estados Unidos da } \\
\text { América }\end{array}$ & $\begin{array}{l}\text { Segurança do paciente na } \\
\text { atenção domiciliar }\end{array}$ & $\begin{array}{l}\text { Descrever o processo usado para desenvolver e avaliar um } \\
\text { sistema de treinamento de simulação virtual interativo para } \\
\text { educar a segurança do paciente domiciliar. }\end{array}$ \\
\hline
\end{tabular}

Fonte: Autores (2020).

O quadro acima tem o intuito de apresentar os países em que os estudos foram desenvolvidos, a temática que foi o foco da simulação, assim como, o objetivo do artigo em análise. Estes dados fazem parte da caracterização dos artigos que fizeram parte desta revisão, tendo em vista que é possível verificar que alguns países têm realizado simulação em temáticas específicas.

Quanto as características dos simuladores, foram identificadas três categorias temáticas sobre o uso da simulação no ensino da Enfermagem, quais sejam: "Simulação virtual - paciente", "Simulação virtual - cenários" e "Simulação virtual imersiva". 
Tabela 1 - Categorias temáticas identificadas sobre simuladores virtuais no ensino da Enfermagem.

\begin{tabular}{l|l}
\hline Categoria temática & Números dos artigos \\
\hline Simulação virtual - paciente & $2,4,6,7,9,10,12,13$ \\
\hline Simulação virtual - cenários & $5,8,11,14$ \\
\hline Simulação virtual imersiva & 1,3 \\
\hline
\end{tabular}

Fonte: Autores (2020).

A tabela acima almeja elucidar as categorias temáticas identificadas nos artigos em estudo, assim como, a prevalência destas. Esta categorização foi necessária tendo em vista a necessidade de conduzir e orientar a discussão dos estudos, para permitir a comparação entre os achados das pesquisas.

\section{Categoria temática: Simulação virtual - paciente}

Os 08 artigos $(57,1 \%)$ identificados nesta categoria, permitiram identificar que a simulação foi realizada na análise de um caso clínico, ou situação clínica, que precisava ser identificada e manejada pelos participantes. Nestes estudos, o exercício da tomada de decisão clínica e raciocínio lógico, foram associadas a conhecimentos de patologias, para que as condutas e intervenções fossem realizadas. Desse modo, permitiu que o estudante pudesse vivenciar o atendimento a um paciente virtual, reforçando conhecimentos e habilidades essenciais para a atuação profissional.

O simulador do estudo de Daher et al. (2020), no artigo 2, trata-se de Paciente Físico-Virtual, que representa a forma de humano, que pode exibir sintomas multissensoriais sutis (por exemplo, temperatura e pulso localizados), uma variedade de cenários (por exemplo, avaliação neurológica, sepse e queimaduras) e uma diversidade de faixas etárias de pacientes (por exemplo, criança, adulto, masculino, feminino, obesos, amputados) e aparência (por exemplo, pele, olhos, cor dos cabelos variados). No referido estudo, foi realizada a análise da ferramenata por profissionais de saúde, e posteriormente com 22 estudantes de enfermagem interagindo com uma criança simulada em dois cenários: sepse e abuso infantil.

No artigo de Padilha et al. (2019), número 4, os participantes foram 44 estudantes de graduação do segundo ano, matriculados em um curso sobre respostas corporais, que buscava o ensino dos sistemas respiratório, cardíaco e urinário. Este estudo utiliza o simulador virtual clínico (Body Interact) que apresenta pacientes virtuais apoiados por um algoritmo fisiológico que recria uma condição de saúde dinâmica que responde a intervenções do usuário.

O estudo número 6, de Olszewski et al. (2018), trata-se de um simulador, usado por 1066 usuários em 70 países, que foi desenvolvido para o aluno praticar a diálise peritoneal em vários cenários clínicos em diversas idades pediátricas e diferentes níveis da doença (insuficiência renal aguda e insuficiência renal crônica), podendo estar associado ou não a fatores associados (sepse, peritonite, malformações congênitas). Este, busca promover uma abordagem sistemática para definir e ajustar a prescrição, complicações mecânicas, anormalidades eletrolíticas e problemas de gerenciamento na assistência específicos do paciente.

Na pesquisa de número 7, de Aebersold et al. (2018), os estudantes realizaram o procedimento de passagem de sonda nasogástrica, e posteriormente, participavam da simulação virtual com a temática de anatomia. A amostra foi de 69 estudantes e os resultados deste estudo, demonstraram a usabilidade e a viabilidade da simulação virtual em anatomia para o treinamento em procedimentos de enfermagem entre estudantes.

O simulador usado no estudo de Slater et al. (2019), número 9, foi com o objetivo de explorar o impacto de uma experiência de treinamento interativo nos elementos morais, emotivos, comportamentais e cognitivos da empatia. Os 54 participantes participaram do programa Virtual Dementia Tour (®), no qual poderiam colocar-se no lugar de um paciente com demência, permitindo um olhar a partir da sua perspectiva. 
O estudo número 10, de Tjoflat et al. (2018), utilizou o simulador $v$ Sim ${ }^{\circledR}$ for Nursing, que trata-se de uma ferramenta americana, construída no idioma inglês. Neste artigo, 65 alunos participaram da simulação virtual que o inseria em um cenário cirúrgico, no qual deveriam realizar cuidados pós-operatórios a uma paciente com complicações.

A ferramenta usada na pesquisa de número 12, de Jütten et al., (2017), trata-se de um simulador com foco na demência, o Into D'mentia, com 142 participantes. Este, assim como o do estudo de número 9, foi desenvolvido para que os cuidadores experimentam como é ter demência, tentando aumentar a empatia pelo paciente com esta condição clínica.

Os autores Aredes et al. (2017), do artigo 13, utilizaram o simulador virtual com foco nos cuidados com a pele na saúde da criança, mais especificamente com a assistência de enfermagem ao neonato prematuro, com o nome de e-Baby: integridade da pele. O simulador apresenta o caso clínico de um prematuro por meio de uma enfermeira virtual que narra os dados relevantes da anamnese e histórico da avaliação clínica. Em seguida, aparece na tela o desafio de avaliar clinicamente o bebê prematuro e promover os cuidados de enfermagem de acordo com os problemas apresentados, e durante a avaliação clínica e definição dos cuidados de enfermagem. O referido estudo, foi validado por um painel de experts.

Para Aredes et al. (2017), artigo 13, o simulador oferece a experiência educativa que simula virtualmente os desafios reais da prática clínica, visando melhor preparo dos estudantes em ambiente seguro e oportunidade de estudo que antecede as ações na unidade neonatal. Dessa forma, os autores ressaltam que pode contribuir como recurso de apoio à prática docente e ao processo de ensino-aprendizagem na perspectiva do estudante, oferecendo uma ferramenta diferenciada para estudo e simulação de situações reais.

A prática no simulador virtual permite que o usuário possa exercitar a tomada de decisão clínica frente a casos clínicos, que vivenciarão na prática de assistência. No simulador de diálise peritoneal ao paciente pediátrico, possibilitava que os alunos avaliassem as condições, manipulassem os recursos e as configuração da máquina, identificassem a necessidade dos ajustes na prescrição e da administração de medicamentos em resposta à avaliação clínica, balanço hídrico e resultados laboratoriais (Olszewski, et al., 2018), artigo 6.

Assemelha-se ao estudo de Padilha et al. (2019), artigo 4, no qual o usuário pode interagir com o paciente virtual por meio de diálogos, monitorando os parâmetros fisiológicos, observação e exame físico, prescrição e/ou análise de exames complementares e prescrição de intervenção e/ou tratamento farmacológico. As respostas e o desenvolvimento do caso clínico são dinâmicos e dependem das decisões tomadas e, a finalização do caso clínico é determinado pela resolução bem-sucedida do cenário ou pela quantidade de tempo decorrido (conforme definido pelo usuário).

No artigo 2, Daher et al. (2020), a interface permite a interação direta com o paciente, o que pode interferir na experiência, avaliação e aprendizado do participante, pois ele precisará tomar as condutas que achar relevantes para a situação identificada.

Para Aredes et al. (2017), artigo 13, a simulação virtual é uma tecnologia educacional que pode ser repetida, permite erro e possibilita novas tentativas com suporte imediato e informativo, e contempla evidências da área da saúde para problemas epidemiologicamente relevantes.

Esta necessidade de aproximação dos casos reais vivenciados na prática clínica, também foi ressaltado no artigo 10 , Tjoflat et al. (2018), no qual a maioria dos estudantes de enfermagem avaliou o cenário clínico virtual em enfermagem cirúrgica como realista e educacional. Entretanto, ressaltou a necessidade de que os docentes estejam preparados para o uso da ferramenta, para que, dessa forma, possam auxiliar os alunos que estiverem com dificuldades em manusear o simulador.

Outros simuladores tinham como foco o desenvolvimento de habilidades dos componentes emocionais, morais, cognitivos e comportamentais, tais como a de empatia, pois colocavam o participante na posição de paciente, e a simulação era conduzida sob esta perspectiva. Foi o caso dos simuladores dos artigos de números 9 e 12, nos quais a experiência foi uma forma de aprendizado que mergulha o participante na experiência imaginada de ter demência e as consequências emocionais 
dessa experiência (Slater, et al., 2019), tendo em vista que oferecer suporte para cuidadores destes pacientes é necessário (Jütten, et al., 2017).

\section{Categoria temática: Simulação virtual - cenários}

Os quatro estudos $(28,5 \%)$ identificados nesta categoria, permitiram identificar que a simulação foi com foco na avaliação de cenários, nos quais almejou contextualizar o estudante em uma perspectiva global de ambiente. Nestes estudos, eram solicitados que os participantes utilizassem habilidades não de avaliação da condição cínica de um paciente, mas de competências sobre a análise de riscos externos, gestão, liderança e relacionamento interprofissional.

O estudo de Polivka et al. (2019), artigo número 5, foi utilizado um Sistema de Treinamento em Simulação Virtual Interativo em Assistência Médica Domiciliar, projetado para treinar profissionais de saúde na assistência domiciliar. O estudo foi realizado com 74 participantes e tinha como foco a identificação de riscos a saúde que possam comprometer a segurança do paciente em um cenário em suas casas.

Para os autores Tschannen et al (2018), artigo 8, utilizaram a simulação virtual no contexto do gerenciamento e saúde. Com a participação de 41 sujeitos, o objetivo da atividade consistiu em inicialmente, recapitular os princípios-chave da gestão, e posteriormente, a participação em três cenários virtuais, exigindo que os participantes usassem habilidades efetivas de liderança.

Na pesquisa de Domingues et al. (2017), número 11, o simulador "Cuidando bem" foi utilizado na disciplina de gerenciamento em Enfermagem, com 11 estudantes, com a temática de segurança do paciente. Ele foi construído em fases, em que cada uma delas trabalha com um Protocolo de Segurança do Paciente: a primeira tem como objetivo a identificação do paciente, a segunda fase lesão por pressão, terceira fase prevenção de quedas, quarta fase cirurgia segura e a quinta fase administração de medicamento.

O simulador do estudo de Darragh et al. (2016), número 14, foi elaborado para o treinamento em gerenciamento de risco e segurança para profissionais de saúde que atuam em cuidados domiciliares. Os cenários foram projetados para fornecer pistas iniciais sobre riscos em potencial e são incorporados às características do cliente e aos riscos existentes. A amostra contou com a participação de 68 sujeitos, para o processo de avaliação.

Para Darragh et al. (2016), artigo 14, os simuladores virtuais facilitam os processos de aprendizado ativo e pensamento crítico que são cruciais para os cuidados domiciliares, pois trata-se de um ambiente imprevisível e precisam resolver problemas para criar soluções para eventos inesperados ou complicados que afetam sua saúde e segurança de seus clientes. Assim, esta experiência da prática vivenciada por simulação, trata-se de um ambiente seguro e controlado de aprendizagem para executarem suas habilidades relacionadas à segurança do paciente (Domingues, et al., 2017), artigo 11.

O treinamento por simulação pode minimizar algumas das barreiras atuais ao sucesso da educação interprofissional, permitindo que os participantes se conectem em várias localizações geográficas, permitindo o amplo uso na educação dos profissionais de saúde (Tschannen, et al., 2018), artigo 8. Dessa forma, os treinamentos virtuais são tão eficazes e envolventes, quanto um treinamento típico (Polivka, et al., 2019), artigo 5.

Desse modo, Darragh et al. (2016), artigo 14, destaca que os treinamentos de saúde e segurança devem enfatizar cenários realistas, soluções flexíveis e atividades independentes de solução de problemas. E para Aebersold et al. (2018), artigo 7, a simulação virtual resultou no aprimoramento de algumas competências, quando comparadas com outras ferramentas.

\section{Categoria temática: Simulação virtual imersiva}

Os dois artigos $(14,2 \%)$ identificados nesta categoria temática, verificou-se que conhecimentos específicos que necessitaram de apoio durante o ensino, foram abordados. Nestes, os alunos puderam exercitar a avaliação ou a técnica, por 
meio de simulação virtual imersiva, identificando quais seriam as suas dificuldades ou lacunas de conhecimento frente a execução de alguma prática.

$\mathrm{Na}$ pesquisa de número 1, de Berg \& Steinsbekk (2020), os autores investigaram a auto-prática individual da abordagem de avaliação primária no atendimento pré-hospitalar, o "VirSam ABCDE", com 18 estudantes. Neste contexto, os resultados indicaram que esta ferramenta pode ser usada como uma maneira prática e envolvente de aprender os fundamentos da abordagem sobre esta temática.

Para os autores Souza-Junior et al. (2020), artigo 3, o simulador "VIDA-Enfermagem v1.0" foi projetado para o ensino das etapas do procedimento de coleta de sangue a vácuo no adulto. O estudo foi avaliado por 15 profissionais da saúde e 15 graduandos de enfermagem em relação aos aspectos visual, interativo, realidade de simulação do movimento, pedagógico e esforço de utilização.

Os simuladores podem ser utilizados por graduandos de enfermagem que estão iniciando no estudo da temática, como uma estratégia a ser combinada com os recursos/habilidades já utilizados no ensino da técnica do procedimento. Assim, proporcionando melhor preparo dos estudantes para o desenvolvimento das competências necessárias para o atendimento ao paciente em estágios supervisionados e, posteriormente, na prática profissional (Souza-Junior, et al., 2020), artigo 3.

\section{Considerações Finais}

O presente estudo permitiu a identificação dos artigos científicos sobre simuladores no ensino da enfermagem, assim como, as áreas temáticas nos quais estes estudos foram produzidos e algumas características destas ferramentas. Destaca-se que estes recursos podem contribuir para a segurança, exercitar a tomada de decisão e o exercício da avaliação de diferentes condições de saúde, inseridos em múltiplos contextos.

Os simuladores são ferramentas dinâmicas, pois a interação dos personagens na simulação possibilita a aprendizagem de maneira mais didática, de modo que podem colocar em prática os conhecimentos da teoria. Dessa forma, podem auxiliar no desenvolvimento do pensamento crítico, reflexivo, que conduzirá a intervenções acuradas e focadas nas reais necessidades do paciente.

O uso de simuladores no ensino não substitui a importância das atividades práticas presenciais com o cuidado direto ao paciente real, pois este trata-se de uma ferramenta que vem a complementar o processo de ensino-aprendizagem em Enfermagem. Pois, existe a multiplicidade de situações que a assistência ao paciente proporciona, e que não seriam passíveis de reprodução por meio de simulações, e que exercitam a capacidade de resolução de problemas.

Como sugestão de estudo futuros, recomenda-se a realização de pesquisas que tenham como foco o uso de simuladores virtuais no ensino da Enfermagem, em diferentes contextos, assim como, a análise da perspectiva do docente sobre a sua aplicação. Além disso, acredita-se ser necessário mais pesquisas que busquem avaliar os aspectos de pensamento crítico dos estudantes de enfermagem durante a utilização destas ferramentas.

\section{Referências}

Aebersold, M., Voepel-Lewis, T., Cherara, L., Weber, M., Khouri, C., Levine, R. \& Tait, A. R. (2018) Interactive Anatomy-Augmented Virtual Simulation Training. Clin Simul Nurs, 15:34-41. doi: 10.1016/j.ecns.2017.09.008.

Antunes, M. (2020). Raciocínio clínico e pensamento crítico dos estudantes de enfermagem: análise a partir da aplicação do simulador virtual 'Health Simulator'. PGIE (Tese de doutorado em Informática na Educação), Universidade Federal do Rio Grande do Sul, Porto Alegre, 2020.

Aredes, N. D. A, Dias, D. M. V., Fonseca, L. M. M., Campbell, S. H., Martins, J. C. A. \& Rodrigues, M. A. (2018). E-baby integridade da pele: inovação tecnológica no ensino de enfermagem neonatal baseado em evidências. Esc Anna Nery, 22(3):e20170424.

Arksey, H. \& O’Malley, L. (2005). Scoping studies: towards a methodological framework. International journal of social research methodology, 8(1):19-32.

Armstrong, R., Burford, B.J., Doyle, J. \& Waters, L. (2011). 'Scoping the scope' of a cochrane review. Journal of Public Health, 33(1):147-50. 
Berg, H. \& Steinsbekk, A. (2020). Is individual practice in an immersive and interactive virtual reality application non-inferior to practicing with traditional equipment in learning systematic clinical observation? A randomized controlled trial. BMC Medical Education, 20:1-10. https://doi.org//10.1186/s12909-02002030-7.

Bez, M., Mello, B., Pinheiro, D. \& Stahnke, F. (2018). Health Simulator: um simulador de casos de estudo para a área da saúde. Revista Observatório, 4(3): 283-306.

Carvalho, E.C.C. (2016). A look at the non-technical skills of nurses: simulation contributions. Rev. Latino-Americana de Enfermagem, $24: e .2791$.

Daher, S., Hochreiter, J., Schubert, R., Gonzalez, L., Cendan, J., Anderson, M., Diaz, D. \& Welch, G. (2020). The Physical-Virtual Patient Simulator: A physical human form with virtual appearance and behavior. Simulation in Healthcare, 15(2):115-121. https://doi .org/10.1097/SIH.0000000000000409.

Domingues, N, A, Tibes, C. M., Dias, J. D., Westin, U. M., Zem. Mascarenhas, S. H., Fonseca, L. M. M. (2017). Virtual simulation by computer on nursing teaching: experience report. Re Enferm UFPI, 6(4):70-74.

Darragh, A. R., Lavender, S., Polivka, B., Sommerich, C. M., Wills, C. E., Hittle, B. A. et al. (2016). Gaming simulation as health and safety training for home health care workers. Clin Simul Nurs, 12 (8), 328e335. http://dx.doi.org/ 10.1016/j.ecns.2016.03.006.

JBI (Joanna Briggs Institute). (2015) Methodology for JBI Scoping Reviews - Joanna Briggs 2015. Australia: JBI; c2015. https://nursing.lsuhsc.edu/JBI/do cs/ReviewersManuals/Scoping-.pdf

Jütten, L. H., Mark, R.E., Janssen, B. W. J. M., Rietsema, J., Dröes, R.M. \& Sitskoorn, M. M. (2017). Testing the effec-tivity of the mixed virtual reality training Into D'mentiafor informal caregivers of people with dementia: Protocolfor a longitudinal, quasi-experimental study. BMJ Open, 21; 7(8): e015702. http://dx.doi.org/10.1136/bmjopen-2016-015702.

Levac, D., Colquhoun, H. \& O'Brien, K.K. (2010). Scoping studies: advancing the methodology. Implement Sci, 5:69. 10.1186/1748-5908-5-69.

Melo, M. et al. (2017). A simulação no ensino da graduação. In: Scalabrini Neto A, Fonseca A, Brandão C. Simulação realística e habilidades na Saúde. Rio de Janeiro: Atheneu, 24-30.

Mendonça, G.U.G. (2019). Construção de um simulador para o ensino da segurança do paciente. MPTIE (Dissertação de Mestrado Profissional). http://bdtd.ibict.br/vufind/Record/UFOR_619c9169ecf94a6b8862a94669f57e07.

Moher,, D., Liberati, A., Tetzlaff, J., Altman, D. G. (2009). Preferred Reporting Items for Systematic Reviews and Meta Analyses: The PRISMA Statement. PLoS Medicine, 6(7), e1000097.

Munn, Z., Peters, M.D.J., Stern, C., Tufanaru, C., McArthur, A. \& Aromataris, E. (2018). Systematic review or scoping review? Guidance for authors when choosing between a systematic or scoping review approach. BMC Med Res Methodol, 18: 143.

Olszewski, A. E., Daniel, D. A., Stein, D. R., McCulloch, M. I., Su, S. W., Hames, D. L. \& Wolbrink, T.A. (2018). Teaching Pediatric Peritoneal Dialysis Globally through Virtual Simulation. Clin J Am Soc Nephrol, 13(6): 900-906.

Padilha, J. M., Machado, P. P., Ribeiro, A., Ramos, J. \& Costa, P. (2019). Clinical Virtual Simulation in Nursing Education: Randomized Controlled Trial. J Med Internet Res, 21(3):e11529.

Pereira, A. S., Parreira, F. J., Shitsuka, D. M., \& Shitsuka, R. (2018). Metodologia da pesquisa científica. UFSM. https://repositorio.ufsm.br/bitstream/ha ndle/1/15824/Lic_Computacao_Metodologia-Pesquisa-Cientifica.pdf?sequence=1.

Polivka, B. J., Anderson, S., Lavender, S. A., Sommerich, C. M., Stredney, D. L. \& Wills, C. E. et al. (2019). Efficacy and Usability of a Virtual Simulation Training System for Health and Safety Hazards Encountered by Healthcare Workers. Games Health J., 8(2):121-128. 10.1089/g4h.2018.0068.

Rodrigues, I. D. C. V., Ferreira, L. B., Lopes, D. C. L., Menezes, H. F. de, Rocha, C. C. T., \& Silva, R. A. R. da. (2020). Simulação realística: aproveitamento e benefícios para o ensino - aprendizagem do raciocínio diagnóstico de enfermagem. Research, Society and Development, 9(7), e553974338. https://doi.org/10.33448/rsd-v9i7.4338

Salvador, P. T. C. O., Bezerril, M. S., Mariz, C. M. S., Fernandes, M. I. D., Martins, J. C. A. \& Santos, V. E. P. (2017). Objeto e ambiente virtual de aprendizagem: análise de conceito. Rev Bras Enferm, 70(3):572-9. http://www.scielo.br/scielo.php?script=sci_arttext\&pid=S0034$71672017000300572 \& \operatorname{lng}=$ pt\&nrm=iso\&tlng=pt.

Silveira, M. S. \& Cogo, A. L. P. (2017). Contribuições das tecnologias educacionais digitais no ensino de habilidades de enfermagem: revisão integrativa. Revista Gaúcha de Enfermagem, 38(2):e66204. https://dx.doi.org/10.1590/1983-1447.2017.02.66204.

Slater P, Hasson F, Gillen P, Gallen A \& Parlour R. (2019). Virtual simulation training: Imaged experience of dementia. Int J Older People Nurs, 14(3): e12243. 10.1111/opn.12243.

Souza-Junior, V. D. de, Mendes, I. A. C., Tori, R., Marques, L. P., Mashuda, F. K. K. \& Hirano, L. A. F. et al. (2020). VIDA-Enfermagem v1.0: realidade virtual imersiva na coleta de sangue a vácuo em adulto. Revista Latino-Americana de Enfermagem, 28:e3263. https://dx.doi.org/10.1590/1518-8345.3685.3263

Tjoflat, I., Brandeggen, T. K., Strandberg, E. S., Dyrstad, D. N. \& Husebo, S. E. (2018). Norwegian nursing students' evaluation of vSim ${ }^{\circledR}$ for Nursing. Adv Simul, 3(10). https://doi.org/10.1186/s41077-018-0070-9.

Tschannen, D., Dorn, R., \& Tedesco, C. (2018). Improving knowledge and behavior of leadership and followership among the interprofessional team. International journal of medical education, 9: 182-188. https://doi.org/10.5116/ijme.5b30.9a84 